\title{
Oscillation of Nonlinear First Order Neutral Differential Equations
}

\author{
Njlaa Issa Tawfiq*
}

Date of acceptance 3/7/2007

\begin{abstract}
:
In this paper, the author established some new integral conditions for the oscillation of all solutions of nonlinear first order neutral delay differential equations. Examples are inserted to illustrate the results.
\end{abstract}

\section{Introduction:}

Consider the first order nonlinear neutral delay differential equation:

$[x(t)+p(t) x(\tau(t))]^{\prime}+q(t) f(x(\sigma(t)))=0$

Subject to the conditions:

(c1) $p(t) \in C[R, R], \tau(t)$ and $\sigma(t)$ are positive nondecreasing continuous functions, such that

$\lim _{t \rightarrow \infty} \sigma(t)=\lim _{t \rightarrow \infty} \tau(t)=\infty$

(c2) $q:\left[t_{0}, \infty\right) \rightarrow R$ is continuous function.

(c3) $f: R \rightarrow R$ is continuous function with $u f(u)>0$ for $u \neq 0$,

and there is a positive constant $M$ such that $f(u) / u^{\alpha} \geq M>0$ where $\alpha$ is a ratio of odd positive integers.

If we let $p(t)=\max \{\tau(t), \sigma(t)\}$ and $T \geq t_{0}$ then by a solution of equation (1), we mean a continuous function $x:\left[t_{0}, \infty\right) \rightarrow R$ such that $x(t)+p(t) x(\tau(t)) \quad$ is continuously differentiable for $t \geq t_{0}$, and $x(t)$ satisfies equation (1) for all $t \geq t_{0}$. A solution of equation (1) is said to be oscillatory if it has arbitrary large zero and nonoscillatory otherwise.
In [4], Gopasamy, Lalli, and Zhang considered the linear equation

$(x(t)+p x(t-\tau))^{\prime}+q(t) x(t-\sigma)=0$

Where $-1<p \leq 0$ and proved that if

$\liminf _{t \rightarrow \infty} \int_{t-\sigma}^{t} q(s) d s>1+p$,

then all solutions of equation (2) are oscillatory. For additional results on the oscillatory behavior of solutions of the linear equation (2), we refer the reader to the monographs by Bainow and Mishev [2], Erbe, Kong, and Zhang [3], and Gyǒri and Ladas [8] as well as the papers of Agarwal and Saker [1], Pahri [15], Saker and Elabbasy [17], Tanaka [18], and Zhou [21] And the references contained therein.

In [5], Graef et al. considered the nonlinear equation

$(x(t)+p x(t-\tau))^{\prime}+q(t) f(x(t-\omega))=0$,

with $f$ nondecreasing, sublinear, $-1<p \leq 0$, and they proved that if $\int_{t_{0}}^{\infty} q(t) d t=\infty$,

then every solution of equation (3) is oscillatory. They also proved a similar result for equation (3) where $f$ is superlinear and $p<-1$.

\footnotetext{
* Department of Mathematics, College Science for Women, University of Baghdad
} 
Section 2, continuous some basic lemmas that are needed to prove my main results, in Section 3, there are some new integral conditions for the oscillation of all solutions of equation (1). This research includes examples to illustrate the main theorem.

\section{Some Basic Lemmas}

In this section, established some lemmas for the case $\alpha=1$. These lemmas will be used to proved the main results.

Lemma 1 Suppose that

$q(t) \geq 0, \tau(t)<t, \sigma(t)<t, p_{2} \geq p(t) \geq p_{1}>1, \sigma(t)<\tau(t) \quad$ for $\quad t \geq t_{0}$ and $\quad \limsup _{t \rightarrow \infty} \int_{t}^{\sigma^{-1}(\tau(t))} q(s) d s>0$

if $x(t)$ is an eventually positive solution of equation (1) then

$\liminf _{t \rightarrow \infty} \frac{z\left(\tau^{-1}(\sigma(t))\right)}{z(t)}<\infty$

Proof Let $x(t)$ is an eventually positive solution of equation (1) for $t \geq t_{0}$ then

$$
z(t)=x(t)+p(t) x(\tau(t))
$$

Which means, $z(t)>0$ and so $z^{\prime}(t) \leq 0$ and hence $z(t)$ is decreasing, we have

$$
\begin{aligned}
& p(t) x(\tau(t))=z(t)-x(t) \\
& z\left(\tau^{-1}(t)\right)=x\left(\tau^{-1}(t)\right)+p\left(\tau^{-1}(t)\right) x(t) \\
& z\left(\tau^{-1}(t)\right) \geq p\left(\tau^{-1}(t)\right) x(t) \geq p_{1} x(t)
\end{aligned}
$$

on the other hand

$$
\begin{aligned}
& p_{2} x(\tau(t)) \geq z(t)-x(t) \\
& p_{2} p_{1} x(\tau(t)) \geq p_{1} z(t)-p_{1} x(t) \geq p_{1} z(t)-z(t)
\end{aligned}
$$

then

$$
x(\tau(t)) \geq \frac{p_{1}-1}{p_{2} p_{1}} z(t)
$$

Or

$$
c(\sigma(t)) \geq \frac{p_{1}-1}{p_{2} p_{1}} z\left(\tau^{-1}(\sigma(t))\right)
$$

from (1) and the last inequality we get

$$
\begin{aligned}
& z^{\prime}(t)+M q(t) x(\sigma(t)) \leq 0 \\
& z^{\prime}(t)+M \frac{p_{1}-1}{p_{2} p_{1}} q(t) z\left(\tau^{-1}(\sigma(t))\right) \leq 0
\end{aligned}
$$

by lemma (1) in [10] follow that

$$
\liminf _{t \rightarrow \infty} \frac{z\left(\tau^{-1}(\sigma(t))\right)}{z(t)}<\infty
$$

\section{Lemma 2}

$$
\begin{array}{cr}
\text { Assume } & \text { that } \\
q(t) \geq 0, \sigma(t)<\tau(t), p_{2} \geq p(t) \geq p_{1}>1 \text { for } t \geq t_{0} \\
\text { if } x(t) \text { is an eventually positive }
\end{array}
$$
solution of equation (1) then

$$
\int_{t}^{\sigma^{-1}(\tau(t))} q(s) d s \leq \frac{p_{1} p_{2}}{M\left(p_{1}-1\right)}
$$

for sufficiently large $t$.

Proof We have $x(t) \geq 0$ and $q(t) \geq 0$, then $z^{\prime}(t) \leq 0$, and $z(t) \geq 0$, if we integrating (6) from $t$ to $\sigma^{-1}(\tau(t))$ we obtain

$$
\begin{aligned}
& z\left(\sigma^{-1}(\tau(t))\right)-z(t)+M \frac{p_{1}-1}{p_{1} p_{2}} \int_{t}^{\sigma^{-1}(\tau(t))} q(s) z\left(\tau^{-1}(\sigma(s))\right) d s \leq 0 \\
& z\left(\sigma^{-1}(\tau(t))\right)-z(t)+M \frac{p_{1}-1}{p_{1} p_{2}} z(t) \int_{t}^{\sigma^{-1}(\tau(t))} q(s) d s \leq 0 \\
& z\left(\sigma^{-1}(\tau(t))\right)+\left(M \frac{p_{1}-1}{p_{1} p_{2}} \int_{t}^{\sigma^{-1}(\tau(t))} q(s) d s-1\right) z(t) \leq 0
\end{aligned}
$$

then

$$
M \frac{p_{1}-1}{p_{1} p_{2}} \int_{t}^{\sigma^{-1}(\tau(t))} q(s) d s-1 \leq 0
$$

(7) follow directly from the last inequality.

\section{Oscillation Results}

In this section, get integral conditions for the oscillation of all solutions of equation (1). Consider the case $\alpha=1$.

$$
\begin{aligned}
& \text { Theorem 1 Assume } \\
& q(t)>0, \sigma(t)<\tau(t), p_{2} \geq p(t) \geq p_{1}>1 ; \\
& \int^{\infty} q(t) \ln \left[\frac{e M\left(p_{1}-1\right)}{p_{1} p_{2}} \int_{t}^{\sigma^{-1}(\tau(t))} q(s) d s\right] d t=\infty
\end{aligned}
$$
that and (4) holds, then every solution of (1) is oscillatory.

Proof For the sake of contradiction we may suppose that $x(t)$ is an eventually 
positive solution of equation (1) then $z(t)>0$ and $z^{\prime}(t)<0$ for $t \geq t_{0}$, from (6) we get

$$
\left.z^{\prime}(t)+M \frac{p_{1}-1}{p_{2} p_{1}} q(t) z\left(\tau^{-1} \sigma(t)\right)\right) \leq 0
$$

using the integral identity $z\left(t_{1}\right)=z(t) e^{-\int_{t_{1}}^{t} \frac{z^{\prime}(s)}{z(s)} d s}$ in (6) we obtain

$-\frac{z^{\prime}(t)}{z(t)} \geq M \frac{p_{1}-1}{p_{2} p_{1}} q(t) e^{\int^{-1 /(\sigma(t))}-z^{\prime}(s)} z(s) d s$

Applying the inequality $e^{r x} \geq x+\frac{\ln (e r)}{r}$ for $x>0$ and $r>0$ to the last inequality we get

$$
\begin{aligned}
-\frac{z^{\prime}(t)}{z(t)} & \geq \frac{M\left(p_{1}-1\right)}{p_{1} p_{2}} q(t) e^{\left.\left(\lambda(t) \frac{1}{\lambda(t)}\right)_{\tau^{-1}(\sigma(t))}^{t} \frac{-z^{\prime}(s)}{z(s)} d s\right)} \\
& \geq \frac{M\left(p_{1}-1\right)}{p_{1} p_{2}} \frac{q(t)}{\lambda(t)}\left[\int_{\tau^{-1}(\sigma(t))}^{t}-\frac{z^{\prime}(s)}{z(s)} d s+\ln (c \lambda(t))\right]
\end{aligned}
$$

where

$$
\lambda(t)=\frac{M\left(p_{1}-1\right)}{p_{1} p_{2}} \int_{t}^{\sigma^{-1}(\tau(t))} q(s) d s
$$

it follows that

$$
-\frac{z^{\prime}(t)}{z(t)} \int_{t}^{\sigma^{-1}(\tau(t))} q(s) d s-q(t) \int_{\tau^{-1}(\sigma(t))}^{t}-\frac{z^{\prime}(s)}{z(s)} d s \geq q(t) \ln \left(\frac{e M\left(p_{1}-1\right)}{p_{1} p_{2}} \int_{t}^{\sigma^{-1}(\tau(t))} q(s) d s\right)
$$

for $T \leq t \leq u, T<\tau^{-1}(\sigma(u))$ we have

$\int_{T}^{u} \int_{t}^{\sigma^{-1}(t(t))} \frac{z^{\prime}(t)}{z(t)} q(s) d s d t-\int_{T}^{u} \int_{\tau^{-1}(\sigma(t))}^{1} q(t)\left(-\frac{z^{\prime}(s)}{z(s)}\right) d s d t \geq \int_{T}^{u} q(t) \ln \left(\frac{e M\left(p_{1}-1\right)^{\sigma^{-1}(t(t))}}{p_{1} p_{2}} \int_{t}^{u} q(s) d s\right) d t \quad(10)$

from the increasing of order of integrating we can conclude that

$$
\begin{aligned}
\int_{T}^{u} \int_{\tau^{-1}(\sigma(t))}^{t} q(t)\left(-\frac{z^{\prime}(s)}{z(s)}\right) d s d t & \geq \int_{T}^{\tau^{-1}(\sigma(u))} \int_{s}^{\sigma^{-1}(\tau(s))} q(t)\left(-\frac{z^{\prime}(s)}{z(s)}\right) d t d s \\
& =\int_{T}^{\tau^{-1}(\sigma(u))} \int_{t}^{\sigma^{-1}(\tau(t))} q(s)\left(-\frac{z^{\prime}(t)}{z(t)}\right) d s d t
\end{aligned}
$$

use the last inequality in (10) we obtain

$\int_{\tau^{-1}(\sigma(u))}^{u}-\frac{z^{\prime}(t)}{z(t)}\left(\int_{t}^{\sigma^{-1}(\tau(t))} q(s) d s\right) d t \geq \int_{T}^{u} q(t) \ln \left(\frac{e M\left(p_{1}-1\right)}{p_{1} p_{2}} \int_{t}^{\sigma^{-1}(\tau(t))} q(s) d s\right) d t$

using lemma 2 we have

$\frac{p_{1} p_{2}}{M\left(p_{1}-1\right)} \int_{\tau^{-1}(\sigma(u))}^{u}-\frac{z^{\prime}(t)}{z(t)} d t \geq \int_{T}^{u} q(t) \ln \left(\frac{e M\left(p_{1}-1\right)}{p_{1} p_{2}} \int_{t}^{\sigma^{-1}(\tau(t))} q(s) d s\right) d t$

or

$\ln \frac{z\left(\tau^{-1}(\sigma(u))\right)}{z(u)} \geq \frac{M\left(p_{1}-1\right)}{p_{1} p_{2}} \int_{T}^{u} q(t) \ln \left(\frac{e M\left(p_{1}-1\right)}{p_{1} p_{2}} \int_{t}^{\sigma^{-1}(\tau(t))} q(s) d s\right) d t$ according to (8) we must have $\lim _{t \rightarrow \infty} \frac{z\left(\tau^{-1}(\sigma(t))\right)}{z(t)}=\infty \quad$ which contradict lemma 1.

\section{Example1}

$[x(t)+(a+\cos (t)) x(t-\pi)]^{\prime}+((1-a)+2 \cos (t)) x\left(t-\frac{3 \pi}{2}\right)=0, \quad t>0$, such that $a>1$, a sample verification yields that the conditions of theorem 1 are met. Hence all solutions of above equation oscillate for instance $x(t)=\cos (t)$ is such solution.

Theorem2 Assume that $q(t) \geq 0, \sigma(t)<\tau(t), 0<p_{2} \leq p(t) \leq p_{1} \leq 1$ and there exists $k>0$ such that

$$
\frac{1}{e} \leq \int_{\tau^{-1}(\sigma(t))}^{t} q(s) d s<k
$$

then every solution of equation (1) is oscillatory.

Proof Suppose that equation (1) has an eventually positive solution $x(t)$, then from (9)

$$
-\frac{z^{\prime}(t)}{z(t)} \geq M \frac{p_{1}-1}{p_{2} p_{1}} q(t) e^{\int^{-1}(\sigma(t))}-\frac{z^{\prime}(s)}{z(s)} d s
$$

let $B(t)=\exp \left(e \int_{\tau^{-1}(\sigma(t))}^{t} q(s) d s\right)$ from

we conclude that $k_{1} \geq B(t) \geq e$ for some $k_{1}>0, t \geq t_{0}$ and we claim that (11) implies that $\lim _{t \rightarrow \infty} \int_{t_{0}}^{t} q(s) d s=\infty$, otherwise $\lim _{t \rightarrow \infty} \int_{t_{0}}^{t} q(s) d s<\infty$.

So we can choose $t_{1} \geq t_{0}$ large enough such that $\int_{t_{1}}^{\infty} q(s) d s<\frac{1}{e}$ which contradict (11) then

$\left.-\frac{z^{\prime}(t)}{z(t)} B(t) \geq \frac{M\left(p_{1}-1\right)}{p_{1} p_{2}} B(t) q(t) e^{\left(\frac{1}{B(t)} B(t)\right.} \int_{\tau^{-1}(\sigma(t))}^{t}-\frac{z^{\prime}(s)}{z(s)} d s\right)$ 
using the inequality $e^{\frac{x}{r}} \geq 1+\frac{x}{r^{2}}, x \geq 0, r \geq 1$

the last inequality will be

$$
\begin{aligned}
& -\frac{z^{\prime}(t)}{z(t)} B(t) \geq \frac{M\left(p_{1}-1\right)}{p_{1} p_{2}} B(t) q(t)\left(1+\frac{B(t)}{B^{2}(t)} \int_{\tau^{-1}(\sigma(t))}^{t}-\frac{z^{\prime}(s)}{z(s)} d s\right) \\
& -\frac{z^{\prime}(t)}{z(t)} B(t) \geq \frac{M\left(p_{1}-1\right)}{p_{1} p_{2}} q(t)\left(B(t)+\int_{\tau^{-1}(\sigma(t))}^{t}-\frac{z^{\prime}(s)}{z(s)} d s\right)
\end{aligned}
$$

for $T \leq t \leq u, T<\tau^{-1}(\sigma(u))$ we get

$\int_{T}^{u}-\frac{z^{\prime}(t)}{z(t)} B(t) d t-\frac{M\left(p_{1}-1\right)}{p_{1} p_{2}} \int_{T}^{u} q(t) \int_{\tau^{-1}(\sigma(t))}^{t}-\frac{z^{\prime}(s)}{z(s)} d s d t \geq \frac{M\left(p_{1}-1\right)}{p_{1} p_{2}} \int_{T}^{u} q(t) B(t) d t$

by interchange the order of the integrating we have

$\int_{T}^{u}-\frac{z^{\prime}(t)}{z(t)} B(t) d t-\frac{M\left(p_{1}-1\right)}{p_{1} p_{2}} \int_{T}^{\tau^{-1}(\tau(u))}-\frac{z^{\prime}(t)}{z(t)} \int_{t}^{\sigma^{-1}(\tau(t))} q(s) d s d t \geq \frac{M\left(p_{1}-1\right)}{p_{1} p_{2}} \int_{T}^{u} q(t) B(t) d t$

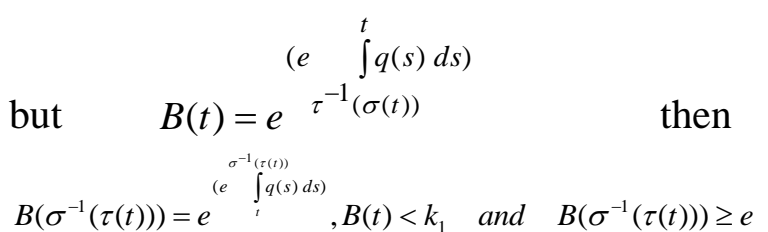
so the last inequality lead to

$k_{1} \int_{T}^{u}-\frac{z^{\prime}(t)}{z(t)} d t-\frac{e M\left(p_{1}-1\right)}{p_{1} p_{2}} \int_{T}^{\tau^{-1}(\sigma(u))}-\frac{z^{\prime}(t)}{z(t)} d t \geq \frac{M\left(p_{1}-1\right)}{p_{1} p_{2}} \int_{T}^{u} q(t) B(t) d t$

where $k_{1} \leq \frac{e M\left(p_{1}-1\right)}{p_{1} p_{2}}$

$\frac{e M\left(p_{1}-1\right)}{p_{1} p_{2}}\left(\int_{T}^{u}-\frac{z^{\prime}(t)}{z(t)} d t-\int_{T}^{\tau^{-1}(\sigma(u))}-\frac{z^{\prime}(t)}{z(t)} d t\right) \geq \frac{e M\left(p_{1}-1\right)}{p_{1} p_{2}} \int_{T}^{u} q(t) d t$

then $\int_{\tau^{-1}(\sigma(u))}^{u}-\frac{z^{\prime}(t)}{z(t)} d t \geq \int_{T}^{u} q(t) d t \quad$ as

$t \rightarrow \infty$ we get $\lim _{t \rightarrow \infty} \frac{z\left(\tau^{-1}(\sigma(t))\right)}{z(t)}=\infty$

which is contradict lemma 1 .

Example 2 Consider the equation $\left[x(t)+\frac{1}{2}\left(1+\sin ^{2}(t)\right) x(t-2 \pi)\right]^{\prime}+\frac{3}{2}\left(1+\sin ^{2}(t)\right) x\left(t-\frac{5 \pi}{2}\right)=0, \quad t>0$, which satisfies all conditions of theorem 2 , therefore each solution of above equation oscillate for example $x(t)=\sin (t)$ is an oscillatory solution.

Theorem 3 Suppose that $q(t) \leq 0, p_{1} \geq p(t) \geq p_{2}>1, \tau^{-1}(\sigma(t))>t, \lim _{t \rightarrow \infty} \tau^{-1}(\sigma(t))=\infty \quad$ for $t \geq t_{0}$

and

$\liminf _{t \rightarrow \infty} M \frac{p_{1}-1}{p_{1} p_{2}} \int_{t}^{\tau^{-1}(\sigma(t))}|q(s)| d s>\frac{1}{e}$

Then every solution of equation (1) is oscillatory.

Proof For the sake of contradiction we may suppose that $x(t)$ is an eventually positive solution of equation (1) then $z^{\prime}(t) \geq 0$ and $z(t)>0$ and we have $z(t)=x(t)+p(t) x(\tau(t)$ $p(t) x(\tau(t))=z(t)-x(t)$ $z\left(\tau^{-1}(t)\right)=x\left(\tau^{-1}(t)\right)+p\left(\tau^{-1}(t)\right) x(t)$ $z\left(\tau^{-1}(t)\right) \geq p\left(\tau^{-1}(t)\right) x(t) \geq p_{1} x(t)$

on the other hand we have $p_{2} x(\tau(t)) \geq z(t)-x(t)$ $p_{1} p_{2} x(\tau(t)) \geq p_{1} z(t)-p_{1} x(t) \geq p_{1} z(t)-z(t)$

$p_{1} p_{2} x(\tau(t)) \geq p_{1} z(t)-z(t)$

$p_{1} p_{2} x(\tau(t)) \geq z(t)\left(p_{1}-1\right)$

then we get

$x(\tau(t)) \geq \frac{p_{1}-1}{p_{1} p_{2}} z(t) \quad$ or $\quad x(\sigma(t)) \geq \frac{p_{1}-1}{p_{1} p_{2}} z\left(\tau^{-1}(\sigma(t))\right)$

from (1) we can get

$z^{\prime}(t)=-q(t) \frac{f(x(\sigma(t)))}{x(\sigma(t))} x(\sigma(t))$

but we have the condition $\frac{f(x)}{x} \geq M$ if we applying it in last equation $-q(t) \frac{f(x(\sigma(t)))}{x(\sigma(t))} x(\sigma(t)) \geq-M q(t) x(\sigma(t))$

then

$z^{\prime}(t) \geq-M q(t) x(\sigma(t))$

$z^{\prime}(t) \geq M|q(t)| x(\sigma(t)) \geq M \frac{p_{1}-1}{p_{1} p_{2}}|q(t)| z\left(\tau^{-1}(\sigma(t))\right)$

$z^{\prime}(t)-M \frac{p_{1}-1}{p_{1} p_{2}}|q(t)| z\left(\tau^{-1}(\sigma(t))\right) \geq 0$

Which is contradict lemma 1.3.2 in [14]. Example 3 Consider the neutral equation $\left[x(t)+\left(3-\frac{\cos (t)}{2}\right) x\left(t-\frac{3 \pi}{2}\right)\right]^{\prime}+(\cos (2 t)-4) x\left(t-\frac{5 \pi}{4}\right)=0, \quad t>0$, all that the conditions of theorem 3 are satisfied, hence each solutions of the above equation oscillate for example $x(t)=\cos (2 t)$ is an oscillatory solution. 
Theorem 4 Assume that

$$
q(t) \leq 0,0 \leq p(t) \leq p<1, \tau(t) \leq t, f
$$

is an increasing function and

$$
\int_{t_{0}}^{\infty}|q(s)| d s=\infty
$$

then all bounded solutions of equation (1) are oscillatory.

Proof We suppose that $x(t)$ is bounded and positive solution of equation (1) for $t \geq t_{0} \quad$ which implies to $z^{\prime}(t) \geq 0, z(t) \geq 0$ that means $z(t)$ is bounded increasing function and we have $z(t) \geq x(t)$ and $z(\tau(t)) \geq x(\tau(t))$ but $z$ is increasing then $z(\tau(t)) \leq z(t)$ and we have too,

$z(t)=x(t)+p(t) x(\tau(t)) \leq x(t)+p x(\tau(t))$

$z(\tau(t)) \leq z(t) \leq x(t)+p x(\tau(t)) \leq x(t)+p z(\tau(t))$

$z(\tau(t)) \leq x(t)+p z(\tau(t))$

$z(\tau(t))-p z(\tau(t)) \leq x(t)$

$(1-p) z(\tau(\sigma(t))) \leq x(\sigma(t))$

$\because f$ increasing we can get

$f((1-p) z(\tau(\sigma(t)))) \leq f(x(\sigma(t)))$

$-q(t) f((1-p) z(\tau(\sigma(t)))) \leq-q(t) f(x(\sigma(t)))$

then from equation (1) and the last inequality we obtain

$$
z^{\prime}(t)+q(t) f((1-p) z(\tau(\sigma(t)))) \geq 0
$$

by integrating this inequality from $t_{0}$ to $t$

$$
z(t)-z\left(t_{0}\right)+\int_{t_{0}}^{t} q(s) f((1-p) z(\tau(\sigma(s)))) d s \geq 0
$$

which implies to

$$
z(t)-z\left(t_{0}\right)+f\left((1-p) z\left(\tau\left(\sigma\left(t_{0}\right)\right)\right)\right) \int_{t_{0}}^{t}|q(s)| d s \geq 0
$$

then if $t \rightarrow \infty$ we get contradiction.

Example 4 Consider the neutral equation $\left[x(t)+\frac{1}{4}(2+\cos (t)) x(t-2 \pi)\right]^{\prime}+\frac{1}{2}(-3-\cos (t)) x(t-2 \pi)=0, \quad t>0$, all that the conditions of theorem 3 are satisfied, hence each solutions of the above equation oscillate for example $x(t)=\cos (t)$ is an oscillatory solution.

Theorem 5 Assume that $q(t) \geq 0, p(t) \geq 0, \tau(t) \leq t, f$ is an increasing function and

$\int_{t_{0}}^{\infty} q(s) d s=\infty$

then all bounded solutions of equation (1) are oscillatory.

Proof Suppose that $x(t)$ is an eventually positive solution of equation (1) then $z^{\prime}(t) \leq 0, z \geq 0$ for $t \geq t_{0}$ and by (1) we have

$$
z^{\prime}(t)=-q(t) f(x(\sigma(t)))
$$

by integrating this equation from $t_{0}$ to $t$ we get

$z(t)-z\left(t_{0}\right)=-\int_{t_{0}}^{t} q(s) f(x(\sigma(s))) d s \leq-f\left(x\left(\sigma\left(t_{0}\right)\right)\right) \int_{t_{0}}^{t} q(s) d s$ $z(t)-z\left(t_{0}\right) \leq-f\left(x\left(\sigma\left(t_{0}\right)\right)\right) \int_{t_{0}}^{t} q(s) d s$

as $t \rightarrow \infty$ we can get $\lim _{t \rightarrow \infty} z(t)=-\infty$ which is contradiction since $z(t)>0$.

Example 5 Consider the neutral equation $[x(t)+(6-\cos (t)) x(t-\pi)]^{\prime}+(5+2 \cos (t)) x\left(t-\frac{3 \pi}{2}\right)=0, \quad t>0$, all that the conditions of theorem 3 are satisfied, hence each solutions of the above equation oscillate for example $x(t)=\cos (t)$ is an oscillatory solution.

\section{References:}

1. Agarwal, R. P. and Saker, S. H.,2001, Oscillation of Solutions to Neutral Delay Differential Equations With Positive and Negative Coefficients Ine J. Differ. equ. Appl.2: 449-465.

2. Bainov, D. D. and Mishev, D. P.,1991, Oscillation Theory for Neutral Differential Equations with Delay, Adam Hilger, New work.

3. Erbe, L. H. Kong, Q. and Zhang, B.G., 1995, Oscillation Theory of Functional Differential Equation, Marcel Dekker, New York

4. Gopalsamy, K., Lalli, B. S. and Zahang, B. G.,1992, Oscillation of Odd Order Neutral Differential Equations, Czech. Math. J. 42:313-323.

5. Graeg,J. R., Grammatikopoulos, M. K. and Spikes, P. W.,1991,Asymptotic and 
Oscillatory Behavior of Solutions of First

Order Nonlinear Neutral Delay Differential Equations, J. Math. Anal. Appl. 155:562-571.

6. Graef, J. R., Grammatikopoulos, M. K. and Spikes, P. W.,1993, Asymptotic Behavior of Nonoscillatory Solutions of Neutral Delay Differential Equations of Arbitrary Order, Nonlinear Anal. 21:2342.

7. Graef, J. R., Savithri, R. and Thandapani, E.,2004, Oscillation of First Order Neutral Delay Differential Equations, EJQTDE, Proc. 7th coll. QTDE.

8. Gyǒri, I. and Ladas, G.,1991, Oscillation Theory of Delay Differential Equations, Clarendon Press, Oxford.

9. Jaros, J. and Kusano, T.,1991, Oscillation Properties of First Order Nonlinear Functional Differential Equations of Neutral Type, Differential Integral Equations 4:425-436.

10. Li, B.,1996, Oscillation of First Order Delay Differential Equations, Proc. Amer. Math. Soc. 124:3729-3737.

11. Li, W. T. and Saker, S. H.,2001, Oscillation of Nonlinear Neutral Delay Differential Equations With Applications, Ann. Polon. Math. 77:39-51.

12. Li, W. T. and Saker, S. H., Oscillation of Neutral Delay Nonlinear Differential Equations, Soochow J. Math., to appear. 13. Mishra, B. B.,2001, On the Qualitative Behavior of Solutions of Neutral Delay
Differential Equations, Ph.D. Thesis, Utkal Univ. India.

14. Issa, Njlaa, 2002, Some Properties of the Oscillatory and Nonoscillatory Solutions of Second Order Linear Neutral Differential Equations, Ms.D. Thesis, Baghdad Univ. Iraq.

15. Parhi, N.,2000, Oscillations of Higher Order Differential Equations of Neutral Type, Czech. Math. J. 50:155-173.

16. Yilmaz, Y. S. and Zafer, A.,2001, Bounded Oscillation of Nonlinear Differential Equations of Arbitrary Order, Czech. Math. J. 51:185-195.

17. Saker, S. H. and Elabbasy, El. M.,2001,Oscillation of First Order Neutral Delay Differential Equations, Kyungpook Math. J. 41:311-321.

18. Tanaka, S., 2000, Oscillatory and Nonoscillatory Solutions of Neutral Differential Equations, Ann. Polon. Math. 73:169-184.

19. Tanaka, S.,2002, Oscillation of Solutions of First Order Neutral Differential Equations, Hiroshima Math. J. 32:79-85.

20. Tang, X. H.,2002,Oscillation for The First Order Superlinear Delay Differential Equations, J. London Math. Soc. 65:115122.

21. Zhou, Y., 2000,Oscillation of Neutral Functional Differential Equations, Acta Math. Hungar. 86:205-212.

$$
\begin{aligned}
& \text { تذبذب المعادلات التفاضلية المحايدة غير الخطية ذات الرتبة الأولى } \\
& \text { *مدرس مساعد/ كلية العلوم للبنات/ قسم الرياضيات نجلاءع عيسى توفيقي" } \\
& \text { نم في هذا البحث: در اسة المعادلة التفاضلية المحايدة غبر الخطية من الرنبة الأولى بالصيغة } \\
& {[x(t)+p(t) x(\tau(t))]^{\prime}+q(t) f(x(\sigma(t)))=0}
\end{aligned}
$$

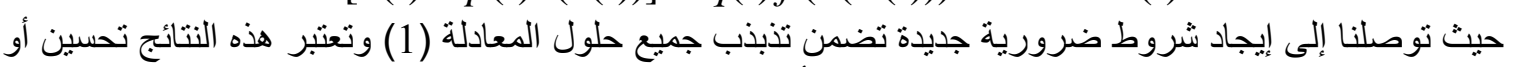

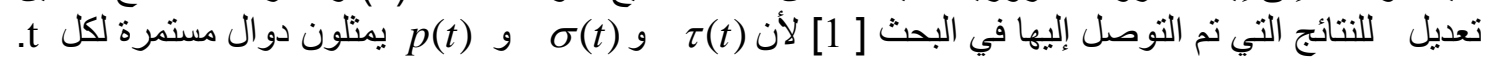

$$
\begin{aligned}
& \text { حيث تضمن البحث } 5 \text { نظريات معززة بأمثلة لتأكيد وجود الحل لهذه المعادلة. لهان. }
\end{aligned}
$$

